\title{
A Vitamina D na Prevenção de Doenças Crónicas: Uma Análise Baseada na Evidência Científica
}

\section{Vitamin D in the Prevention of Chronic Diseases: An Evidence Based Analysis}

\author{
António VAZ-CARNEIRO $\triangle^{1,2}$ \\ Acta Med Port 2017 May;30(5):351-353 - https://doi.org/10.20344/amp.9176
}

Palavras-chave: Doença Crónica/prevenção e controlo; Suplementos Nutricionais; Vitamina D

Keywords: Chronic Disease/prevention \& control; Dietary Supplements; Vitamin D

A vitamina $D$ existe na dieta com lacticínios e alimentos fortificados e é sintetizada na pele sob a acção da radiação ultravioleta. Existem duas formas principais desta hormona: a vitamina $D_{2}$ (ergocalciferol) e a vitamina $D_{3}$ (colecalciferol).

O principal papel da vitamina $D$ é no metabolismo do cálcio, promovendo a mineralização óssea e, por consequência, a sua principal utilização tem sido na prevenção da osteoporose em doentes de risco, assim como no tratamento de fracturas osteoporóticas. Para além de receptores ósseos, existem também receptores da vitamina $D$ em vários outros órgãos e tecidos, o que levanta a questão da sua utilização em contextos preventivos de doenças crónicas, incluindo o seu impacto na mortalidade global. ${ }^{1}$

Neste editorial iremos discutir o uso da vitamina $D$ fora das indicações ósseas, apresentado a evidência mais recente e de melhor qualidade sobre o rastreio da vitamina $\mathrm{D}$ e a sua utilização como medida preventiva de doenças crónicas.

\section{O conceito de risco em medicina}

Estatisticamente falando, o risco define-se como sendo a probabilidade que algo inesperado aconteça. Neste sentido, se alguém compra um bilhete do Euromilhões 'arrisca-se' a ganhar uma soma importante. Por outro lado, o conceito de risco é em medicina sempre negativo, isto é, está ligado a um evento prejudicial para a saúde. Quando afirmamos que fumar é um factor de risco para doenças cardiovasculares, respiratórias e oncológicas, o que estamos a dizer é que o tabagismo pode aumentar a incidência deste tipo de doenças.

No contexto do risco, há sempre três vertentes: a primeira é a sua quantificação, a segunda é a sua comunicação e a terceira é a sua gestão.

A maneira mais rigorosa de determinar se um factor de risco aumenta a incidência de uma qualquer doença é realizar um estudo em que o investigador divide a amostra de pessoas sem a doença em causa em dois grupos: um é exposto ao risco e o outro não. Passado algum tempo, qualquer diferença nas taxas de incidência de doença pode ser atribuída ao factor de risco. Dado que este tipo de estudos não é eticamente aceitável (não é possível estudar dano potencial em pacientes), lança-se mão de estudos observacionais de dois tipos: de coorte e/ou caso-controlo. As medidas de associação nestes casos são o risco relativo (nos estudos de coorte) ou o odds ratio (nos caso-controlo). Um valor destas medidas acima da unidade $(2,5,10$, etc.) informa-nos que um paciente com um determinado factor de risco tem 2, 5, 10 vezes mais probabilidades de vir a ter a doença, do que um outro paciente em que o referido factor de risco está ausente.

Por outro lado, em termos de gestão do risco o factor crucial é a sua dimensão: com efeito, quando pensamos no tratamento de um factor de risco (ou, melhor dizendo, na sua modulação) temos de levar em linha de conta que, quanto mais alto é o seu valor, mais a intervenção que propomos utilizar é eficaz. Por outras palavras, quanto mais alto for o risco de base, mais eficaz é a terapêutica. Isto porque, numa análise de benefício/dano, o benefício da intervenção é muito superior ao seu potencial dano e, portanto, deve ser oferecida a todos os doentes elegíveis. É o caso do tratamento do cancro.

Inversamente, no caso de riscos de base de pequenas dimensões, a intervenção pode ter um efeito negativo, já que o benefício é modesto e, portanto, a análise de benefício/dano pode pender para o lado do dano. É o caso do tratamento de níveis de colesterol ligeiramente elevados em doentes jovens, em que os efeitos adversos podem ser mais importantes que o pequeno benefício esperado na incidência de doença cardiovascular.

Deve-se recomendar um rastreio de massas para detecção de níveis baixos de vitamina $D$ ?

Alguns estudos observacionais têm estabelecido correlações entre baixos níveis séricos de 25-hidroxivitamina D3 (25(OH)D3) e um conjunto de doenças crónicas, pelo que se tem decretado que a hipovitaminose $D$ é "uma pandemia" necessitando de detecção e tratamento. ${ }^{2}$

Com base nesta conviç̧ão, tem-se proposto rastreio de massas para detecção de hipovitaminose D.

\footnotetext{
1. Centro de Estudos de Medicina Baseada na Evidência. Faculdade de Medicina. Universidade de Lisboa. Lisboa. Portugal.

2. Centro Colaborador Português da Rede Cochrane Iberoamericana. Lisboa. Portugal.

$\square$ Autor correspondente: António Vaz-Carneiro. avc@medicina.ulisboa.pt

Recebido: 08 de maio de 2017 - Aceite: 08 de maio de 2017 | Copyright $\odot$ Ordem dos Médicos 2016
} 
Um primeiro problema desta abordagem é que a consistência do doseamento sérico de $25(\mathrm{OH}) \mathrm{D}_{3}$ é problemática, devido há existência de múltiplos testes apresentando variação dos resultados entre métodos (com variações de $10 \%-20 \%$ ) e entre os diversos laboratórios. Junta-se a isto o facto de não haver um standard internacionalmente aceite.

Um segundo problema é epidemiológico: a maior parte dos peritos aceita que níveis inferiores a $50 \mathrm{nmol} / \mathrm{L}$ (20 ng/ $\mathrm{mL}$ ) se associam a problemas ósseos, baseados num conjunto muito vasto de estudos sugerindo que a este nível a saúde do osso está comprometida. ${ }^{3} \mathrm{O}$ respectivo tratamento consistiria na ingestão de pelo menos 600 - $800 \mathrm{UI}$ de vitamina $D$ por dia, na tentativa de atingir o referido nível sérico (não entrando em linha de conta com eventual exposição solar). Como referido num recente editorial, ${ }^{4}$ a ideia que a totalidade da população deverá idealmente ter valores superiores a $20 \mathrm{ng} / \mathrm{mL}$ para conseguir uma boa saúde óssea ignora que mais de $98 \%$ da população precisa de menos, devido a variação individual de necessidades com uma distribuição normal abaixo daqueles valores (daí os $97,5 \%$, dois desvios padrão). Estas definições, que provêm do Institute of Medicine dos Estados Unidos da América, calculam que - neste contexto - apenas uma pequena percentagem dos pacientes necessite de suplementação vitamínica. Na prática, para que toda a população atingisse os tais $20 \mathrm{ng} / \mathrm{mL}$ (baseada na associação entre a ingestão referida e o nível sérico que se pretende alcançar - que se designa como recommended dietary allowance) - seria necessária uma ingestão regular muito significativa de vitamina D pela grande maioria da população, com os potenciais impactos clínicos (efeitos adversos tipo nefrolitíase, fracturas, etc.) e - não menos importante - financeiros.

Finalmente, a não existência de estudos comparando benefícios e riscos em pacientes rastreados versus não rastreados - o que seria a evidência mais importante para suportar esta decisão - levanta questões sobre a desejabilidade do rastreio de massas desta hormona. Esta medida preventiva, baseada em níveis de corte inapropriados (20 $\mathrm{ng} / \mathrm{mL}$ ), poderia levar a suplementação em pessoas sem qualquer necessidade terapêutica (o chamado sobretratamento).

\section{Vitamina D como prevenção de doenças crónicas}

Que evidência científica existe de suporte à utilização da vitamina $\mathrm{D}$ como medida preventiva em doenças crónicas, especialmente cardiovasculares e/ou oncológicas?

Para resposta a esta questão, pesquisámos - utilizando o Ovid - a Medline e a Embase de 1996 até ao mês de abril de 2017, tendo pré-seleccionado apenas ensaios clínicos, revisões sistemáticas e meta-análises e normas de orientação clínica, analisando o uso de vitamina D como medida preventiva crónica de doença cardiovascular e oncológica (assim como outras doenças crónicas e a mortalidade global). Obtiveram-se 240 estudos e, para este editorial (que não é uma revisão sistemática da literatura), seleccionaram-se os que foram simultaneamente de melhor qualidade e os mais recentes.

Os dados mais úteis e sólidos na resposta a esta questão provêm de revisões sistemáticas com ou sem meta-análises de ensaios clínicos analisando o impacto da vitamina $D$ na incidência de doenças cardiovasculares e cancro (e também diabetes, doença pulmonar obstrutiva crónica e outras). Também se devem analisar dados de estudos observacionais, mas estes têm menor valor porque têm tendência a aumentar artificialmente a dimensão dos benefícios.

Um dos estudos mais recente e de melhor qualidade neste contexto é uma revisão de revisões (uma revisão de revisões sistemáticas - designada como 'umbrela review' é uma revisão não de ensaios clínicos, mas sim de revisões sistemáticas analisando a mesma intervenção terapêutica. Nesse sentido, é ainda mais abrangente do que as revisões sistemáticas individuais que a compõem.) procurando responder à questão do efeito da suplementação da dieta com vitamina $D$ em múltiplos resultados (outcomes) clínicos. ${ }^{5}$ Os autores apenas procuraram identificar dois tipos de estudos: revisões sistemáticas e meta-análises comparando a administração de vitamina $D$ com placebo, ou vitamina D com outro composto (por ex. cálcio), ou esse composto isolado; e estudos observacionais analisando níveis séricos populacionais de vitamina D. Foram incluídos indicadores de doenças oncológicas, cardiovasculares, ósseas, autoimunes, infecciosas, metabólicas (e outras), em todo o tipo de populações. Excluíram-se estudos com exposição à radiação ultravioleta, os que tinham a vitamina $D$ como outcome em si e estudos de prevalência de hipovitaminose em populações específicas. Foram seleccionadas para análise final 107 revisões sistemáticas de literatura e 74 meta-análises de estudos observacionais com concentrações plasmáticas de vitamina $\mathrm{D}$, assim como 87 meta-análises de ensaios clínicos aleatorizados com suplementação de vitamina $D$ (mediana do $n^{\circ}$ de estudos foi 4 e de sujeitos estudados foi de 446). Das 57 meta-análises que não se sobrepuseram em termos de ensaios clínicos, os outcomes relevantes para a nossa análise indicaram uma redução pouco significativa de doença cardiovascular [2 estudos, redução do risco relativo $(\mathrm{RRR})=5 \%$; intervalo de confiança (IC) $95 \%=-5$ a 14] e a mortalidade global, que diminuiu muito discretamente com a vitamina $D_{3}(9$ estudos, $n=12$ 824; $A R R=9 \%$; IC 95\% = -2 a 18) mas que aumentou ligeiramente com a vitamina D2 [8 estudos, $n=17$ 079; aumento do risco relativo $(A R R)=4 \%$; IC 95\% $=-3$ a 11]. Os autores reportaram que a associação entre vitamina $D$ e cancro foi apenas analisada através de estudos observacionais, ou seja, não existem ensaios clínicos estudando esta questão. De resto, os autores conseguiram fazer esta comparação em 14 outcomes, documentando as diferenças entre os resultados emparelhados e afirmando que não existe evidência convincente do benefício entre os dois tipos de estudos. As conclusões globais são que estes resultados são inconsistentes para provar benefício e que, portanto, não podem suportar recomendações universais para administração de vitamina $\mathrm{D}$ em doenças crónicas. 
Uma outra fonte de informação relevante pode ser encontrada numa revisão sistemática da Cochrane recentemente publicada que incluiu 56 ensaios clínicos com 95286 participantes analisando os efeitos na mortalidade global da suplementação de vitamina $D$ em doentes saudáveis ou com doenças crónicas em fases estáveis. ${ }^{6}$ Os autores detetaram uma diminuição da mortalidade muito modesta em idosos com a utilização de vitamina $D_{3}$ $\left(R R=0,97 ;\right.$ IC $95 \% 0,94$ a 0,$\left.99 ; p=0,02 ; I^{2}=0 \%\right)$. As outras formas de vitamina $D$ não tiveram qualquer impacto, e a terapêutica foi acompanhada de riscos de hipercalcemia e nefrolitíase. Os autores concluem que os dados não são suficientes - quer em qualidade quer em quantidade - para poderem servir de apoio à decisão de utilizar a vitamina $\mathrm{D}$ como medida preventiva da mortalidade global (baseados nesta revisão, ter-se-ia de tratar 150 doentes durante cinco anos para evitar uma morte).

Por outro lado, outra revisão sistemática publicada simultaneamente correlacionou níveis séricos de vitamina $D$ e/ou a sua suplementação com a mortalidade global em 73 estudos de coorte e 22 ensaios clínicos, tendo identificado que a vitamina $D_{3}$ pode reduzir ligeiramente a mortalidade, mas que estatisticamente o benefício da suplementação não difere da do placebo. ${ }^{5}$ Estes resultados foram confirmados na revisão sistemática de suporte à Norma de Orientação Clínica da USPSTF, em que se verificou uma ligeira diminuição da mortalidade, mas apenas em doentes institucionalizados (8 estudos, $\mathrm{RR}=0,93$; IC $95 \% 0,73$ a 1,18).

Finalmente, a European Society for Clinical and Economic Aspects of Osteoporosis and Osteoarthritis (ESCEO) publicou em maio um relatório científico em que, após revisão cuidada da evidência de melhor qualidade disponível, afirma que embora estudos seccionais cruzados e de coorte associem níveis séricos de vitamina $\mathrm{D}_{3}$ inferiores a $20 \mathrm{ng} / \mathrm{mL}$ a uma maior incidência de doenças crónicas (até com uma relação dose-resposta positiva), não se justificam quaisquer recomendações de administração de vitamina $D$, já que meta-análises de ensaios clínicos e ensaios individuais não confirmam estes achados, são de dimensões modestas, não estudam todos os indicadores relevantes e não reportam efeitos adversos. ${ }^{7}$

\section{CONCLUSÃO}

Nos últimos anos tem havido um interesse muito marcado sobre os efeitos da vitamina $D$ na saúde, com publicação de um número elevado de estudos (a dimensão da literatura científica sobre a vitamina $D$ é quase igual à de todas as outras vitaminas combinadas).

Apesar de toda esta investigação, e como acontece tantas vezes em medicina, não é possível emitir recomendações claras e bem fundamentadas acerca dos benefícios e riscos da administração de vitamina $\mathrm{D}$ como medida preventiva de doenças crónicas.

Em termos práticos, e baseada na melhor evidência científica, é, no entanto, possível concluir que:

- A vitamina $D$ tem um papel bem definido na prevenção e no tratamento de doenças ósseas.

- O rastreio de massas para detecção de níveis baixos de vitamina $D$ não é recomendável, pelas seguintes razões:

- Os testes laboratoriais para a detecção de vitamina D são pouco fiáveis, por apresentarem variações técnicas significativas e resultados inconsistentes de doente para doente (e até no mesmo doente);

- A noção que o nível normal de vitamina $D$ sérica deve ser superior a $20 \mathrm{ng} / \mathrm{mL}$ é desmentido pelo facto da maior parte dos pacientes ter requerimentos inferiores aos necessários para atingir aquele valor;

- A taxa de doentes/pacientes com níveis considerados baixos é significativa, mas não há evidência sólida de benefício com a suplementação vitamínica

- Não existe consenso generalizado sobre a dosagem ideal para a administração de vitamina $D$ num contexto preventivo, até porque os níveis séricos e as dosagens variam de doença para doença.

- Entre nós, um simples passeio de manga curta de 6-7 minutos durante o dia no verão, ou 7 - 40 minutos de área maximamente exposta no inverno é suficiente para a manutenção de níveis adequados de vitamina $\mathrm{D}$.

- A vitamina $D$ não parece ter um benefício consistente na diminuição da mortalidade global, exceto em doentes institucionalizados com baixos níveis séricos de vitamina $\mathrm{D}_{3}$.

- Não existe evidência científica de boa qualidade que suporte a administração de vitamina $D$ para prevenção de doenças crónicas, pelo que esta medida deve ser desencorajada.

meta-analyses of observational studies and randomised trials. BMJ. 2014;348:g2035.

6. Bjelakovic G, Gluud LL, Nikolova D, Whitfield K, Wetterslev J, Simonetti $\mathrm{RG}$, et al. Vitamin $\mathrm{D}$ supplementation for prevention of mortality in adults. Cochrane Database Syst Rev. 2014;1: CD007470.

7. Cianferotti L, Bertoldo F, Bischoff-Ferrari HA, Bruyere O, Cooper $\mathrm{C}$, Cutolo $\mathrm{M}$, et al. Vitamin $\mathrm{D}$ supplementation in the prevention and management of major chronic diseases not related to mineral homeostasis in adults: research for evidence and a scientific statement from the European society for clinical and economic aspects of osteoporosis and osteoarthritis (ESCEO). Endocrine. 2017;56:245-61. 